\title{
The Immunity Induced by Recombinant Spike Proteins of SARS Coronavirus in Balb/c Mice
}

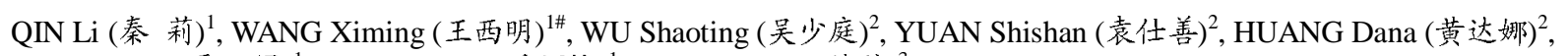 \\ LEI Mingjun (雷明军 $)^{1}$, PAN Huirong (潘辉榕) ${ }^{1}$, LIN Qiping (林绮萍) ${ }^{3}$ \\ ${ }^{1}$ Department of Biochemistry and Molecular Biology, Tongji Medical College, Huazhong University of Science and Technology, \\ Wuhan 430030, China \\ ${ }^{2}$ Shenzhen Center for Disease Control and Prevention, Shenzhen 518020, China \\ ${ }^{3}$ Veterinarian College, South China University of Agriculture, Guangzhou 510000, China
}

\begin{abstract}
Summary: The immune effect of two recombinant protein fragments of spike protein in severe acute respiratory syndrome coronavirus (SARS CoV) was investigated in Balb/c mice. Two partial spike gene fragments S1 (322-1464 bp) and S2 (2170-2814 bp) of SARS coronavirus were amplified by RT-PCR, and cloned into pET-23a prokaryotic expression vector, then transformed into competent Escherichia E.coli BL21 (DE3)(pLysS) respectively. Recombinant proteins were expressed and purified by $\mathrm{Ni}^{2+}$ immobilized metal ion affinity chromatography. The purified proteins mixed with complete Freund adjuvant were injected into Balb/c mice three times at a two-week interval. High titer antibody was detected in the serum of immunized Balb/c mice, and mice immunized with $\mathrm{S} 1$ protein produced high titer $\mathrm{IgG} 1, \mathrm{IgG} 2 \mathrm{a}, \mathrm{IgG} 2 \mathrm{~b}$ and $\mathrm{IgG} 3$, while those immunized with $\mathrm{S} 2$ protein produced high titer IgG1, IgG2a, but lower titer IgG2b and IgG3. Serum IFN- $\gamma$ concentration was increased significantly but the concentrations of IL-2, IL- 4 and IL-10 had no significant change. And a marked increase was observed in the number of spleen CD8+ T cells. The results showed that recombinant proteins of SARS coronavirus spike protein induced hormonal and cellular immune response in Balb/c mice.

Key words: severe acute respiratory syndrome; coronavirus; spike protein; IgG; IgG1; IgG2a; IgG2b; IgG3; IL-2; IL-4; IL-10; IFN- $\gamma$; T lymphocyte subset
\end{abstract}

The severe acute respiratory syndrome coronavirus (SARS-CoV) emerged in Asia as a highly aggressive pathogen that can be lethal in adults and the elderly ${ }^{[1]}$. Spike glycoprotein on the surface of the virus particle mediates the attachment of the virus to the cell surface receptors and induces the fusion of the viral and cellular membrane ${ }^{[2,3]}$. It is also a major target of the immune response to coronavirus. According to the epitope analysis and structure of $S$ protein, two parts of the $S$ protein (108-488aa, 723-938aa), called S1 and S2 here, were cloned and purified to investigate the immune effect in $\mathrm{Balb} / \mathrm{c}$ mice ${ }^{[4]}$.

\section{MATERIALS AND METHODS}

\subsection{Materials}

SARS-CoV culture supernatant and the serum of convalescent SARS patients were provided by Dr. HE Yaqing and Dr. LIU Jianjun (Shenzhen CDC, China). E. coli BL21 (DE3)(pLysS) and expression vector pET-23a were purchased from Novagen Inc. (Germany), E.coli JM109 was purchased from Amersham Biosciences Inc. (Sweden). Six-eight week Female Balb/c mice (SPF grade) were provided by Laboratory Animal Center of

QIN Li, female, born in 1976, Doctor in Charge

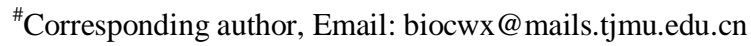

Zhongshan University (China). pMD18-T vector, one-step RT-PCR kit, Taq DNA polymerase, restricted endonuclease, agarose gel DNA fragment recovery kit and DNA standard marker were the products of Takara Biotechnology Co. Ltd. (Japan). Viral RNA purification kit was purchased from Qiagen Biotechnology Inc. (Germany). Protein standard marker was the product of NEB Inc. (USA). HRP-conjugated goat anti-mouse and HRP-conjugated goat anti-human IgG, mouse IL-2, IL-4, IL-10, IFN- $\gamma$ ELISA kit were purchased from Pierce Inc. (USA).

\subsection{Construction of Expression Vector of S1 and S2 Genes}

The genome RNA of SARS-CoV was purified with viral RNA purification kit. The reverse transcription polymerase chain reaction (RT-PCR) primers of S1 gene were 5'-GGATCCAACAACAAGTCACAGTCGGT-3', and 5'-GTCGACGCCAGTAGTGGTGTAAAAA-3', and those of S2 gene were 5'-AGGATCCATCTGCGGAGA TTCTACTGA-3', and 5'-AGTCGACAGCATTCTGGT TAACAACGT-3'. The amplification reaction was performed under the following conditions: incubation at $50^{\circ} \mathrm{C}$ for $30 \mathrm{~min}$, preliminary denaturation at $94^{\circ} \mathrm{C}$ for 2 min, 30 cycles of $94^{\circ} \mathrm{C}$ for $30 \mathrm{~s}, 53^{\circ} \mathrm{C}$ for $30 \mathrm{~s}$, and $72^{\circ} \mathrm{C}$ for $6 \mathrm{~min}$. The purified products were ligated with pMD-18T vector and transformed into E. coli JM109 competent bacteria. The positive bacterial colonies named pMD18T-S1 and pMD18T-S2 were identified by colony PCR and digestion with restriction endonuclease, 
and sequenced by Takara Inc. pMD18T-S1 and pMD18T-S2 plasmids were purified and digested with restricted endonuclease $\mathrm{Bam} \mathrm{H} \mathrm{I}$ and $\mathrm{Sal} \mathrm{I}$, then ligated to pET-23a plasmid digested with the same endonuclease respectively and transformed to $E$. coli BL21 (DE3)(pLyS). The positive colonies were identified by PCR and digested with restriction endonuclease.

\subsection{Expression, Purification and Western Blot Assay} of Recombinant Protein

The positive colonies were incubated in LB culture medium containing ampicillin $(100 \mathrm{mg} / \mathrm{L})$ at $37^{\circ} \mathrm{C}$ with vigorous shaking until $\mathrm{A} 600$ had reached 0.6-0.9, then induced with $1 \mathrm{mmol} / \mathrm{L}$ isopropyl $\beta$-D-thiogalac topyranoside (IPTG) at $37^{\circ} \mathrm{C}$ for $6 \mathrm{~h}$. The bacteria were harvested and lysed at $4^{\circ} \mathrm{C}$, and the supernatant was applied to a column containing Ni-IDA resin. The recombinant protein was eluted with $250 \mathrm{mmol} / \mathrm{L}$ imidazole. The purified protein was separated by $15 \%$ SDS-PAGE and transferred to a NC membrane. Then the membrane was blocked in $5 \%$ skimmed milk/phosphate-buffered saline for $2 \mathrm{~h}$ at $37^{\circ} \mathrm{C}$, and then incubated with $1: 50 \mathrm{di}-$ luted serum of convalescent SARS patients for $2 \mathrm{~h}$ at $37^{\circ} \mathrm{C}$, which was followed by washing with PBS. The blot was then incubated with 1:5000 diluted horseradish peroxidase (HRP)-conjugated goat anti-human antibody. Signals were detected with DAB staining.

\subsection{Immunization Procedures}

Six to eight weeks old BALB/c mice were maintained under standard laboratory conditions. Recombinant $\mathrm{S} 1$ and $\mathrm{S} 2$ antigen $(50 \mu \mathrm{g})$ emulsified in Freund's complete adjuvant (FCA) was injected subcutaneously $(100 \mu \mathrm{L})$ three times at a two-week interval. Control animals received PBS or CFA only. The serum was obtained from mouse tail vein before every vaccination, and the mice were killed 7 weeks after the first vaccination.

\subsection{Analysis of the Antibody Response of Mice by ELISA}

96-well plates were coated with $100 \mu \mathrm{L}$ of purified antigen solution $(50 \mu \mathrm{g} / \mathrm{mL})$ diluted in $50 \mathrm{mmol} / \mathrm{L}$ carbonate-bicarbonate buffer ( $\mathrm{pH}=9.6)$. The titer of the mice sera was detected by indirect enzyme linked immunosorbent assay (ELISA) and the absorbance $(A)$ was measured at $450 \mathrm{~nm}\left(A_{450}\right)$. The titer of the sample was determined when the $A_{450}$ value of the sample was twice greater than the $A_{450}$ value of control sera. Isotypes of serum $\operatorname{IgG}$ were detected by ELISA described above except that the secondary antibody was HRP-conjugated goat anti-mouse IgG1, IgG2a, IgG2b and IgG3.

\subsection{Detection of Spleen T-lymphocyte Subsets by Flow Cytometry}

The mice were killed and the spleens were removed to obtain single cell suspension. According to the instructions of two color flow cytometric analysis kit, $1 \times 10^{6}$ cells were incubated with FITC- or PE-conjugated monoclonal antibody recognized CD3, CD4 and CD8, and analyzed on a FACSCalibur flow cytometer calibrated (Becton Dickinson, USA). The percentage of CD4 positive and CD8 positive T cells were determined.

\subsection{Analysis of the Cytokine Concentration of Im-} mune Sera

The concentrations of IFN- $\gamma$, IL-2, IL-10, IL-4 in the serum of mice were detected according to the in- structions of mouse IL-2, IL-4, IL-10, IFN- $\gamma$ ELISA kit.

\subsection{Statistical Analysis}

Numerical tata were presented as $\bar{x} \pm s_{\bar{x}}$. The data were analyzed with one-way ANOVA followed by Fish's least-significance-difference (LSD) test. Two group comparisons were analyzed by Student's $t$-test. The level of significance was set at $P<0.05$.

\section{RESULTS}

\subsection{Construction of Recombinant Expression Vector}

$\mathrm{S} 1$ and S2 genes were cloned and their sequence matched the GenBank sequence. The recombinant plasmid was identified by PCR and digested with the restriction enzymes BamH I and Sal I. As shown in fig. 1,1143 bp and 645 bp bands were observed in the agarose gel.

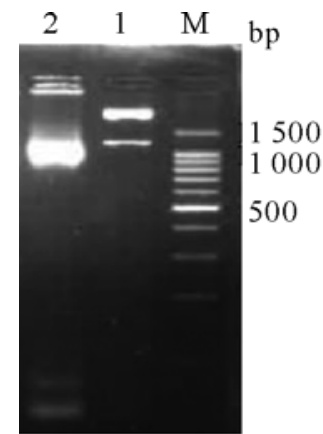

A

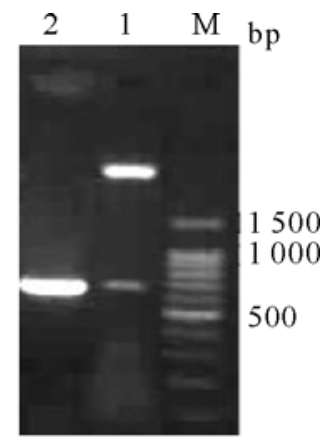

B
Fig. 1 Identification of pET-S1 (A) and pET-S2 (B) by PCR and digestion with Bam H I and Sal I 1: Digested with BamH I and Sal I; 2 Clony PCR product; M: DNA Marker

\subsection{Expression, Purification and Western Blot Assay of Recombinant Protein}

The recombinant bacteria expressed target protein observed in SDS-PAGE gels. S1 and S2 proteins were purified and the molecule weight was about $25 \mathrm{kD}$ (1 $\mathrm{kD}=0.9921 \mathrm{ku}$ ) and $43 \mathrm{kD}$ respectively (fig. 2). The purified protein could be identified by the sera of convalescent SARS patients. As shown in fig. 3, there were two stained fragments in the pyroxylin membrane.
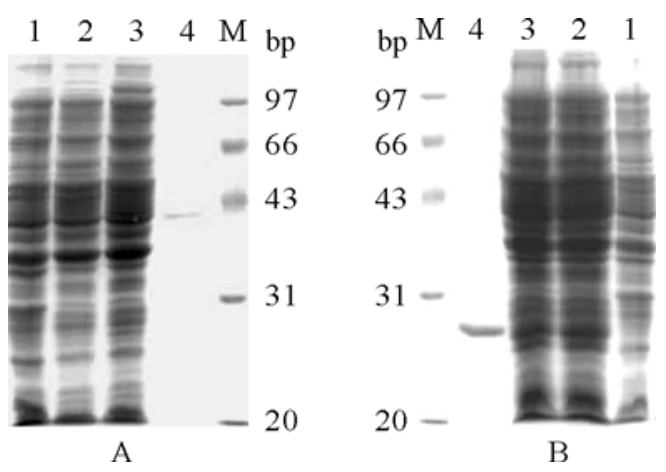

Fig. 2 SDS-PAGE of recombinant pET-S1 (A) and pET-S2 (B)

1: E. coli BL21 (pET-23a); 2: pET-S1/S2 transformants; 3: pET-S1/S2 transformants induced with IPTG;

4: Purified S1/S2; M: Marker 

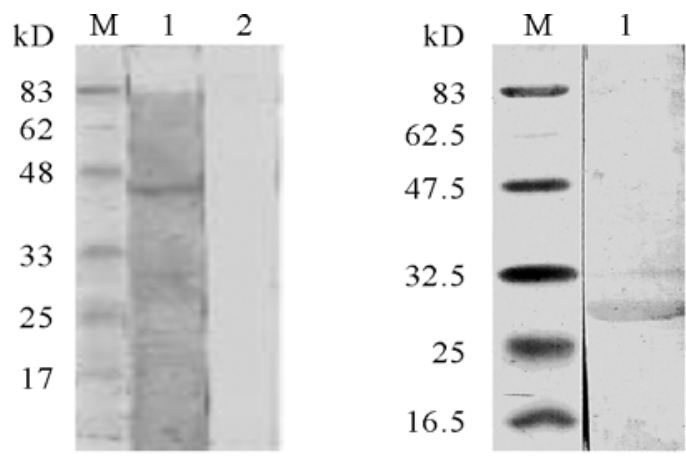

Fig. 3 Western blot of purified S1 protein from the sera of patients with SARS

1: Serum of SARS patients; 2: Normal serum;

$\mathrm{M}$ : Prestained protein marker

\subsection{Specific Antibody Levels and the Isotype of IgG}

The titers of antibody were notably enhanced after every boosting of immunization with the antibody with titer more than 12800 after three times of immunization. The 4 isotypes of $\mathrm{IgG}$ were induced in $\mathrm{S} 1$ and $\mathrm{S} 2$ protein immunized mice. The titers of IgG1, IgG2a, IgG2b and IgG3 were 1:12 800, 1:12 800, 1:12 800 and 1:1600 in S1 protein immunized mice and those were 1:12 800, 1:12 800, 1:6400 and 1:400 in S2 protein immunized mice respectively.

\subsection{T Lymphocyte Subsets in Spleen of Mice}

The CD8+ T cells were increased significantly in S1 and S2 groups as compared with CFA and PBS groups, but the CD4+ T cells did not change remarkably. However the CD4+ $\mathrm{T}$ cells in CFA group were increased significantly as compared with PBS group (table 1).

Table 1 The T lymphocyte subsets in spleen of mice ( $n=6)$

\begin{tabular}{lrc}
\hline Groups & \multicolumn{1}{c}{ CD4+ } & CD8+ \\
\hline PBS & $27.52 \pm 2.50$ & $7.1 \pm 1.47$ \\
CFA & $31.16 \pm 2.33^{*}$ & $7.8 \pm 1.33$ \\
S1 & $33.3 \pm 5.95$ & $9.56 \pm 0.99^{*}$ \\
S2 & $27.52 \pm 5.79$ & $11.8 \pm 3.15^{*}$ \\
\hline
\end{tabular}

${ }^{*} P<0.05$ as compared with PBS group

\subsection{The Cytokine Concentration of Immune Sera}

As shown in table 2, the concentration of IL-2 in CFA, S1 and S2 groups was increased as compared with PBS group, but no significant difference was found between S1/S2 groups and CFA group. However, there was no significant difference between the control groups and recombinant protein immune groups in the concentrations of IFN- $\gamma$, IL-4 and IL-10.

Table 2 The cytokine concentrations of immune sera $(n=6)$

\begin{tabular}{lcccl}
\hline Groups & IFN- $\gamma$ & IL-2 & IL-10 & IL-4 \\
\hline PBS & $1.42 \pm 1.52$ & $6.5874 \pm 4.95$ & $53.07 \pm 9.17$ & $215.33 \pm 73.31$ \\
CFA & $15.41 \pm 15.41$ & $10.6248 \pm 4.11^{*}$ & $108.43 \pm 159.92$ & $249.20 \pm 247.67$ \\
S1 & $46.285 \pm 95.91$ & $10.6248 \pm 4.88^{*}$ & $68.43 \pm 40.53$ & $305.22 \pm 83.44$ \\
S2 & $134.11 \pm 347.68$ & $12.1945 \pm 5.54^{*}$ & $108.922 \pm 107.07$ & $213.31 \pm 206.92$ \\
\hline \multicolumn{5}{c}{$P<0.05$ as compared with PBS group }
\end{tabular}

\section{DISCUSSION}

SARS-CoV is an enveloped RNA virus, which contains several structural proteins. Among these proteins, the spike protein is divided into two subdomains, $\mathrm{S} 1$ and $\mathrm{S} 2$, which have distinct functions. S1 mediates binding to host cell receptors and S2 mediates viral-host cell fu$\operatorname{sion}^{[5,6]}$. Spike protein is also the main protective antigen inducing the generation of neutralizing antibodies, and it can be detected in infected cell culture supernatants with antisera from SARS patients ${ }^{[7,8]}$. The epitopes specific for SARS virus had been screened and two fragments, with many epitopes, were chosen to immunize mice to evaluate the immune responses to them.

Both $\mathrm{S} 1$ and $\mathrm{S} 2$ proteins were expressed in $E$. coli using a pET-23 expression vector after the IPTG induction. Purified S1 and S2 recombinant proteins were recognized by the sera of SARS patients by Western blotting, indicating that recombinant $\mathrm{S} 1$ and $\mathrm{S} 2$ proteins retained its antigenicity. The immune responses to antigen include the generation of specific antibodies and cellular immune response. The mice immunized with S1 and S2 proteins produced specific IgG. The titers reached the maximum at the sixth week after three vaccinations and the maximum titer remained for 14 weeks. Both Th1 and Th2 lymphocytes can help B lymphocyte produce specific antibody. In mice Th1 lymphocytes secrete Th1 type cytokines such as IL- 2 and IFN- $\gamma$ to induce the specific cellular immunization and to promote the production of $\operatorname{IgG} 2 \mathrm{a}, \operatorname{IgG} 2 \mathrm{~b}$ and $\mathrm{IgG} 3$, while Th2 lymphocytes secrete Th2 type cytokines such as IL-4, -5, -6, -10 to promote the production of IgG1. Recombinant S1 protein elicited high titers of $\operatorname{IgG} 1, \operatorname{IgG} 2 \mathrm{a}$ and $\operatorname{IgG} 2 \mathrm{~b}$ and $\mathrm{S} 2$ protein elicited high titers of IgG1 and IgG2a in mice. The results showed that $\mathrm{S} 1$ and $\mathrm{S} 2$ proteins elicited both Th1 and Th2 type immune responses. The sera cytokine analysis showed that only IL-2 concentration was increased as compared with PBS group, but no significant difference was found as compared with CFA group, implying the recombinant protein has no influence on the sera Th1 and Th2 type cytokines secretion. In this study, we only detected the cytokines in sera 7 weeks after the first immunization. The noticeably elevated percentage of CD8+ T lymphocyte subsets indicated that the recombinant protein elicited the definite cellular immune responses, but the mechanism needed further study.

In conclusion, the recombinant $S 1$ and $S 2$ proteins induced definite hormonal and cellular responses in mice, and the protein as a candidate subunit vaccine still needs further study.

\section{REFERENCES}

1 World Health Organization. Consensus document on the epidemiology of severe acute respiratory syndrome (SARS). WHO (http: // www.who.int/csr/sars/en /WHO concensus.pdf)(2003)

2 Marra M A, Jones S J M, Astell C R et al. The genome sequence of the SARS-associated coronavirus. Science, 2003,300:1399-1404

3 Rota P A, Oberste M S, Monroe S S et al. Characterization of a novel coronavirus associated with severe acute respiratory syndrome. Science, 2003,300:1394-1398

4 Bioinformatics Center of Shanghai Life Science Institute 
of Chinese Academy of Sciences. Primary analysis of epitope of SARS coronavirus. http://www.biosino.org/ feidian/ SARS-biaoweifenxi.htm(2003)

5 Wong $\mathrm{S} \mathrm{K}, \mathrm{Li}$ W, Moore M J et al. A 193-amino-acid fragment of the SARS coronavirus S protein efficiently binds angiotensin-converting enzyme 2. J Biol Chem, 2004,279(5):3197-3201

6 Simmons G, Reeves J D, Rennekamp A J et al. Characterization of severe acute respiratory syndrome-associated coronovirus (SARS-CoV) spike glycoprortein-mediated viral entry. Proc Nati Acad Sci, 2004,101(12):4240-4245

7 Sui J H, Li W H, Murakami A et al. Potent neutralization of severe acute respiratory syndrome (SARS) coronavirus by a human $\mathrm{mAb}$ to $\mathrm{S} 1$ protein that blocks receptor association. Proc Nati Acad Sci, 2004,101(8):2536-2541

8 Zhou T, Wang H, Luo D et al. An exposed domain in the severe acute respiratory syndrome coronavirus spike protein induces neutralizing antibodies. J Virol, 2004, 78:7217-7226

(Received May 24, 2006) 